\title{
CARACTERÍSTICAS DE PERSONALIDAD DEL ESPECTRO ESQUIZOFRÉNICO Y LOCUS DE CONTROL EN PADRES NO AFECTADOS DE PACIENTES ESQUIZOFRÉNICOS
}

\author{
BEATRIZ CAPARRÓS ${ }^{1}$, NEUS BARRANTES-VIDAL ${ }^{2}$ y JORDI OBIOLS ${ }^{2}$ \\ ${ }^{1}$ Universidad de Gerona \\ ${ }^{2}$ Universidad Autónoma de Barcelona
}

(Aceptado en abril de 2001)

La investigación en el campo de la esquizofrenia se ha dirigido, en los últimos años, a la detección de marcadores de vulnerabilidad en sujetos con riesgo al trastorno. Esta vulnerabilidad denominada "esquizotipia" puede ser identificada en sujetos clínicamente no afectados con una disposición heredada a desarrollar esquizofrenia. El objetivo de este trabajo se ha centrado en el estudio de las características de personalidad del espectro esquizofrénico como marcadores de vulnerabilidad y en el estudio de la variable locus de control en padres no afectados de pacientes esquizofrénicos. Los datos han sido obtenidos de 26 parejas de padres no afectados de pacientes con esquizofrenia y de 26 parejas de padres normales de sujetos normales, un total de 104 sujetos han sido evaluados. Los instrumentos utilizados en la evaluación han sido el Oxford-Liverpool Inventory of Feelings and Experiences (Mason, Claridge y Jackson, 1995), el Cuestionario de Evaluación IPDE (Loranger, 1988), el Symptom Checklist Revised (Derogatis, 1977) y el Multidimensional Health Locus of Control (Wallston, Wallston y DeVellis, 1974). Los resultados muestran que el grupo de padres de pacientes esquizofrénicos presentan más rasgos de esquizotipia negativa, más características del trastorno paranoide y por evitación de la personalidad, más manifestaciones psicopatológicas en general y un mayor locus de control interno.

Palabras clave: Esquizotipia, esquizofrenia, personalidad, familiares, locus de control.

\section{Personality traits of schizophrenic spectrum and locus of control in non-affected parents of schizophrenic patients}

In the last years, the schizophrenia research has been addressed to the detection of vulnerability markers in subjects at risk. This vulnerability called "schizotypy" can be identified in clinically non-affected subjects with a genetic disposition to the disorder. The main objective of this work has been the study of personality traits of schizophrenic spectrum as a vulnerability markers, and the study of locus of control variable in non-affected parents of schizophrenic patients. Data has been obtained from 26 couples of non-affected parents of schizophrenic patients and from 26 couples of normal parents of normal subjects, a total of 104 subjects have been evaluated. The Oxford-Liverpool Inventory of Feelings and Experiences (Mason, Claridge and Jackson, 1995), the Cuestionario de Evaluación IPDE (Loranger, 1988), the Symptom Checklist Revised (Derogatis, 1977) and the Multidimensional Health Locus of Control (Wallston, Wallston and DeVellis, 1974) have been used to test the subjects. The results has shown that parents of schizophrenic group have more negative schizotypal traits, more traits of the paranoid and avoidance personality disorders, more general psychopathological manifestations and a higher internal locus of control.

Key words: Schizotypy, schizophrenia, personality, relatives, locus of control.

Correspondencia: Beatriz Caparrós, Departamento de Psicología, Universidad de Girona, Plaça Sant Domènec, 9, 17071 Girona, Teléfono: 972418353 / 972418 346, Fax. 972418 345, Correo-e: beatriz.caparros@udg.es

\section{INTRODUCCIÓN}

Uno de los primeros objetivos de la investigación sobre la etiología de la esquizo- 
frenia ha sido el estudio de la transmisión genética del trastorno. Los estudios de familias, de adopción, de gemelos y de sujetos de riesgo elevado han proporcionado la evidencia del componente genético que posee la esquizofrenia (Kendler, Gruenberg y Tsuang, 1985; Winokur, Scharfetter y Angst, 1985; McGue, Gottesman y Rao, 1986; Tienari et al., 1989; Marcus, Hans, Auerbach y Auerbach, 1993; Erlenmeyer-Kimling et al., 1995; Cardno y McGuffin, 1996; Varma, Zain y Singh, 1997). Aunque el modo de transmisión del trastorno es todavía desconocido, se sabe que los patrones de herencia no se ajustan a modelos mendelianos sencillos, ya que son más complejos y de carácter multifactorial.

Hasta la fecha, el mayor riesgo de padecer esquizofrenia viene determinado por la presencia de esquizofrenia en algún familiar; esta probabilidad de tener esquizofrenia correlaciona también con el grado de proximidad del sujeto con los familiares afectados por ella, es decir, el riesgo es más elevado si un paciente tiene un familiar de primer grado que padece esquizofrenia, que si el afectado por el trastorno es un familiar de segundo o tercer grado.

Los estudios de familias no sólo se han centrado en la investigación del riesgo para la esquizofrenia en familiares de personas afectadas por la enfermedad, sino también en el riesgo de padecer otros trastornos cualitativamente similares pero de menor gravedad, los llamados trastornos del espectro esquizofrénico (Kendler y Gruenberg, 1984; Kendler, Masterson, Ungaro y Davis, 1984; Kendler et al., 1993, Onstad, Skre, Edvardsen, Togersen y Kringlen, 1991; Kety et al. 1994, Maier, Lichtermann, Minges y Reinhard, 1994). Entre estos trastornos podemos destacar los trastornos paranoide, esquizotípico y esquizoide de la personalidad.

A pesar de lo anteriormente mencionado, la realidad muestra que la mayor parte de los esquizofrénicos no tiene antecedentes genéticos aparentes. Ambas evidencias no tienen por qué contradecirse. Tal como postularon Zubin $\mathrm{y}$ Spring (1977) en su teoría de la vulnerabilidad al estrés, los individuos que desarrollan esquizofrenia poseen una predisposición genética a ella, pero es necesaria la interacción entre esta vulnerabilidad y determinados factores ambientales para que hace que se manifieste el trastorno.

Los síndromes familiares no psicóticos similares a la esquizofrenia fueron identificados con el término "esquizotipia» por Rado en 1953. Este autor abrevió el término "fenotipo esquizofrénico» en «esquizotipia». Con este concepto intentó describir los síntomas/rasgos observables en los sujetos con una disposición heredada a desarrollar esquizofrenia. Rado propuso que existía una deficiencia en la capacidad para sentir satisfacción en los sujetos esquizotípicos, es decir, una ausencia de la capacidad de experimentar placer que comportaría una fuerza motivacional deficiente y una incapacidad para organizar acciones intencionadas. De esta deficiencia fundamental surgen los síntomas de anhedonia, miedo y desorganización, la exacerbación de los cuales puede llevar a los síntomas más graves de la esquizofrenia.

A pesar de que Rado fue el primero en acuñar el término de "esquizotipia», fue la descripción, la racionalización teórica y los programas de investigación desarrollados por Meehl $(1962,1989)$ los que marcaron el inicio del estudio actual del trastorno esquizotípico. Meehl propuso un defecto neural integrativo como la única consecuencia fenotípica directa producida por una mutación genética. Esto se refería a una alteración en algunos parámetros de funciones celulares particulares, que se podían manifestar o no en el funcionamiento de sistemas más generales del Sistema Nervioso Central (SNC). Este defecto neural integrativo, 
que Meehl bautizó como «hipocrisia», era el único del que se podía hablar apropiadamente como innato, según el autor. La historia de aprendizaje social sobre individuos "esquizotáxicos» (individuos que presentaban hipocrisia) daría como resultado una organización de la personalidad que el autor, siguiendo a Rado, llamó "esquizotipia». La esquizotipia se caracterizaría por errores cognitivos, anhedonia, ambivalencia y aversión interpersonal.

En los últimos años, el término esquizotipia ha sido utilizado por algunos autores de manera sinónima al de "vulnerabilidad a la esquizofrenia». Siguiendo a Holzman (1982), la vulnerabilidad se refiere a "una variación perceptible, palpable y mensurable en la estructura o función que representa una predisposición a un proceso específico patológico». Por tanto, la vulnerabilidad per se no es lo mismo que el trastorno, ya que es detectable previamente al inicio del trastorno. Las características de la vulnerabilidad representan una condición necesaria pero no suficiente para el desarrollo del trastorno. Esta concepción del término "vulnerabilidad» es muy parecida a la de "tendencia" utilizada por Meehl y otros autores. En el caso de la esquizofrenia, la vulnerabilidad implicaría la necesidad de estresores ambientales para que se manifestase la expresión del trastorno, es decir, una persona puede ser vulnerable a la esquizofrenia y no desarrollar nunca el trastorno en su forma más manifiesta, aunque sí puede manifestarlo en algún grado y de manera sutil.

Existe una amplia evidencia que apoya la conceptualización de una tendencia latente en la esquizofrenia (Kendler et al., 1985). La psicopatología esquizotípica estaría incluida en esta tendencia latente. La esquizotipia está unida, probablemente por vía genética, a la esquizofrenia. Puede que la influencia más evidente que ayuda a establecer la unión entre la fenomenología esquizotípica y la clínica esquizofrénica venga dada por los estudios de familias y de adopción, que encuentran una proporción más elevada de rasgos y trastornos esquizotípicos entre los familiares biológicos de esquizofrénicos que en otros grupos (Kendler et al., 1991, Grove et al., 1991, Clementz, Gorve, Katsanis y Iacono, 1991). Se ha confirmado, también, la existencia de una vulnerabilidad para la esquizofrenia «no expresada» (Lenzenweger y Loranger, 1989) por tanto la vulnerabilidad puede existir sin que se den marcadas manifestaciones fenotípicas, es decir manifestaciones de vulnerabilidad más sutiles como pueden ser déficit neuropsicológicos o rasgos de personalidad esquizotípica.

Una variable poco abordada en la investigación sobre vulnerabilidad a la esquizofrenia en familiares de pacientes esquizofrénicos ha sido el locus de control (LOC). El LOC se ha investigado en pacientes esquizofrénicos relacionado con los esfuerzos de afrontamiento y las estrategias autoprotectoras como factores importantes en la prevención de la psicosis (Cash y Stack, 1973, Böker y Brenner, 1983).

El término locus de control se refiere a la expectativa sobre la instancia que es responsable de las consecuencias de la propia conducta. La variable procede de la teoría del Aprendizaje Social (Rotter, Chance y Phares, 1972). En sus inicios el locus de control se entendió como una dimensión bipolar, en uno de cuyos extremos estaría el externalismo, o creencia en que la obtención del refuerzo está más allá del control del sujeto, y en el extremo opuesto, el internalismo, o creencia en que los refuerzos dependen de las conductas realizadas. Desde un punto de vista dimensional, Levenson (1973) añade un factor más y propone los siguientes: expectativas de control interno, expectativas de controles externos 
por personas más poderosas y expectativas de controles externos sujetos a la suerte.

Algunos estudios han demostrado que el LOC contribuye a la salud y a la calidad de vida (Evans, 1997). La sensación o creencia de control no sólo facilita la adaptación del individuo a una situación estresante sino que también parece tener un efecto positivo en la salud.

El único estudio hallado que investiga la variable $L O C$ en pacientes esquizofrénicos y sus familiares es el de Böker, Brenner y Würgler (1989). Los resultados indicaron que los pacientes con vulnerabilidad mostraron un menor LOC interno que los familiares no vulnerables. Los familiares con vulnerabilidad mencionaban más las influencias de las capacidades que las de los esfuerzos, mientras que los familiares sin vulnerabilidad consideraban tan importantes los esfuerzos como las capacidades y atribuían el LOC externo de la misma manera al poder de los otros que a la suerte.

El objetivo principal de este trabajo se ha centrado en el estudio de las características de personalidad como marcadores de vulnerabilidad a la esquizofrenia en padres no afectados de pacientes esquizofrénicos, es decir en sujetos que ya han superado la edad de riesgo al trastorno, comparados con padres normales de sujetos normales. La mayoría de estudios se han centrado en hijos de pacientes esquizofrénicos y en familiares adultos. Este último grupo ha sido en la mayoría de los casos heterogéneo, formado básicamente por padres y hermanos. Este hecho puede llevar a una confusión de los resultados ya que éstos han sido casi siempre globales y no han diferenciado los patrones de respuesta de los hermanos (que se pueden encontrar en edad de riesgo para el trastorno) por una parte y de los padres por otra. Las características de los sujetos de este estudio permiten estudiar estas variables sin la influencia de factores de confusión, como puede ser la misma sintomatología esquizofrénica y los efectos de la medicación o de la institucionalización. Así mismo, estos sujetos ya han sobrepasado la edad de riesgo y por tanto las características que puedan identificarse corresponderán a rasgos de vulnerabilidad y no se confundirán con alteraciones "precursoras" del trastorno. Sobre la base de la literatura revisada, se espera hallar rasgos de personalidad esquizotípica y de trastornos del espectro esquizofrénico diferentes entre los padres de los pacientes con esquizofrenia y en los padres controles normales, mostrando los primeros más características en la dirección de la personalidad definida como esquizotípica. Así mismo se espera hallar diferencias en la variable locus de control, mostrando los padres de los pacientes un menor locus interno.

\section{METODOLOGÍA}

\section{Sujetos}

Los sujetos que han formado parte de estudio son 26 parejas de padres no afectados de pacientes esquizofrénicos y 26 parejas de padres normales de sujetos normales de la provincia de Girona y Barcelona. Las edades de los padres y madres de pacientes esquizofrénicos oscilaban entre 43 y 78 años $(56,5 \pm$ $8,21)$, las edades de los padres del grupo control oscilaban entre 45 y 74 años $(57,63 \pm 7,69)$; las medias de edad entre ambos grupos no se diferenciaban significativamente. Respecto al nivel cultural de los sujetos del grupo de padres de esquizofrénicos, 29 padres $(55,8 \%)$ habían realizado estudios primarios, 15 $(28,8 \%)$ estudios secundarios y $8(15,4 \%)$ estudios universitarios. En el grupo control 33 padres $(63,5 \%)$ habían realizado estudios primarios, $16(30,8 \%)$ estudios 
secundarios y $3(5,8 \%)$ estudios universitarios. Los requisitos de inclusión en el grupo de padres de pacientes esquizofrénicos fueron: 1) tener un hijo/a diagnosticado de esquizofrenia, 2) ausencia de enfermedad mental en todos y cada uno de los progenitores, es decir, ningún padre o madre ha sido diagnosticado, tratado o ingresado por ninguna patología mental, y 3) presentar un nivel intelectual y de alfabetización suficiente como para poder entender y responder de manera correcta las pruebas administradas. Los criterios de inclusión para formar parte del grupo control fueron: 1) no tener ningún hijo/a con trastorno psiquiátrico, 2) no presentar historia familiar de enfermedad mental, 3) no haber sido diagnosticados, ingresados o tratados de ninguna enfermedad mental y 4) presentar un nivel intelectual y de alfabetización suficiente como para poder entender y responder de manera correcta las pruebas administradas.

\section{Instrumentos}

1. Se elaboró un cuestionario de datos personales para obtener información sobre las características demográficas de edad, sexo, estudios realizados y profesión. Se preguntaba también por el padecimiento de enfermedades físicas o mentales por las que el sujeto hubiese sido tratado o ingresado, por algún tipo de medicación que estuviese tomando, por la enfermedad física o mental de algún familiar por la que hubiese sido tratado o ingresado, por el número de hijos con esquizofrenia y por el tiempo que hacía que los hijos habían sido diagnosticados del trastorno.

2. La evaluación de la esquizotipia psicométrica se llevó a cabo mediante el Oxford-Liverpool Inventory of Feelings and Experiences (O-LIFE; Mason,
Claridge y Jackson, 1995; versión española de Gutiérrez et al., 1998). El OLIFE es un cuestionario autoadministrado que consta de 120 items con opción de respuesta «Sí» o «No»; está formado por cuatro escalas que corresponden a los cuatro factores identificados en la esquizotipia: a) Experiencias inusuales: este factor contiene $\mathbf{3 0}$ items relacionados con percepciones aberrantes y alucinatorias, y pensamiento mágico; los items son consistentes con los síntomas positivos de la psicosis; b) Desorganización cognitiva y Ansiedad Social: contiene 24 items que describen dificultades en la atención, concentración y toma de decisiones, juntamente con una incapacidad en la intencionalidad, trastornos del humor y ansiedad social; c) Anhedonia introvertida: contiene 27 items que describen la incapacidad de sentir placer y disfrutar de situaciones sociales y de una amplia gama de otras actividades. Este rasgo indica una cierta aversión a la intimidad emocional y física, y pone énfasis en la independencia y soledad; d) Impulsividad noconformista: contiene 23 items que describen características de deshinibición y de impulsividad, relacionada con la violencia, las conductas abusivas y temerarias.

3. Los rasgos de trastornos de personalidad se evaluaron mediante el Cuestionario de Evaluación IPDE, módulo DSM-IV que forma parte del Examen Internacional de los Trastornos de Personalidad, módulo DSM-IV patrocinado por la OMS. EL IPDE está basado en el Personality Disorder Examination de Loranger (1988). Se utilizó la versión española de la OMS (1996). El cuestionario en sí no es un instrumento de diagnóstico y generalmente se pasa previamente a la realización de la entrevista para descartar aquellos casos en los que es poco probable 
que padezcan un trastorno de personalidad o el trastorno de personalidad que interese evaluar. En este estudio se utilizó como una medida que nos proporcionó una estimación de determinadas alteraciones en cada uno de los trastornos que evalúa y no se utilizó como diagnóstico clínico. El IPDE es una prueba autoadministrada que consta de 77 items, con opción de respuesta Verdadero o Falso, relacionados con los trastornos de personalidad del DSM-IV: trastorno paranoide de la personalidad ( 7 items), esquizoide ( 7 items), esquizotípico (9 items), histri6nico (8 items), antisocial (7 items), narcisista ( 9 items), límite (9 items), obsesivo-compulsivo ( 8 items), por dependencia ( 8 items) y por evitación (8 items).

4. Para la evaluación de la psicopatología general se utilizó el Symptom Checklist-Revised (SCL-90-R; Derogatis, 1977), adaptado al castellano por González de Rivera et al. (1989). Esta es una escala autoadministrada que presenta una lista de 90 items sobre problemas y situaciones por las que el sujeto se puede haber sentido afectado en la última semana. La escala evalúa 9 dimensiones psicopatológicas (somatización, obsesividad-compulsividad, sensibilidad interpersonal, depresión, ansiedad, hostilidad, ansiedad fóbica, ideación paranoide y psicoticismo) y 7 escalas adicionales (apetito negativo, apetito positivo, trastornos del sueño-conciliación, mantenimiento, despertar prematuro- pensamientos de muerte y culpabilidad. También proporciona un Índice General de Síntomas (GSI), el Total de Síntomas Positivos (PST) y el Nivel de Trastorno de los Síntomas Positivos (PSDI).

5. Se utilizó el Multidimensional Health Locus of Control (MHLC, Wallston, Wallston y DeVellis, 1974) para la eva- luación de las creencias sobre la causa de los refuerzos para las conductas relacionada con la salud, es decir si ésta es principalmente interna, debida a la suerte 0 al poder de los otros. Esta escala autoadministrada consta de 18 items referidos al mantenimiento o a la pérdida de la salud. Cada ítem tiene 6 posibilidades de respuesta que van desde $" 1=$ completamente de acuerdo» a « $6=$ completamente en desacuerdo». La escala proporciona tres puntuaciones correspondientes a los tres factores que evalúa: locus de control interno, suerte y poder de los otros. Cada factor está formado por seis items y cada uno de estos items únicamente puntúa en un factor en la dirección del mismo. Así, cuando el sujeto está de acuerdo con el enunciado puntúa 1 punto, lo cual quiere decir que puntuaciones bajas indican la dirección de la escala, por ejemplo puntuaciones bajas en LOC interno indican un mayor internalismo.

\section{Procedimiento}

El procedimiento de la selección y evaluación fue el siguiente:

1. El primer paso fue identificar sujetos con esquizofrenia, diagnosticados según criterios DSM-III-R y DSM-IV por psiquiatras expertos. Una vez identificados los pacientes con esquizofrenia, se contactó telefónicamente (en algunos casos, personalmente) con los padres para explicarles el objetivo del estudio a grandes rasgos y en qué consistiría. En este momento se pedía colaboración de ambos progenitores.

2. Fueron evaluadas aquellas parejas en las que ambos miembros estaban de acuerdo en participar en el estudio. En la primera sesión se les explicó a los padres que el objetivo del estudio se 
basaba en la vulnerabilidad a los trastornos mentales y en qué consistiría su colaboración; así mismo se les informó del anonimato y confidencialidad de los resultados obtenidos. En esta primera sesión se preguntaba por los datos personales para poder descartar aquellos sujetos que no cumplian los requisitos para formar parte del estudio, y contestaban el SCL-90 para descartar los padres que, aunque no hubiesen sido nunca diagnosticados ni tratados por algún trastorno mental, pudiesen presentar alteraciones psicopatológicas importantes. Aquellos padres que cumplían los requisitos eran evaluados en posteriores sesiones en las medidas de esquizotipia, características de trastornos de personalidad y locus de control. El grupo control lo formaron padres normales de sujetos que no habian padecido y no padecían un trastorno mental. La selección de este grupo, por las características que debía tener y con el objetivo de que fuese equiparable en edad y nivel cultural a los padres de los pacientes, no se realizó al azar sino que se contactó con diferentes padres que cumplían los requisitos previos y que voluntariamente accedieron a formar parte de la investigación. El procedimiento a seguir para el grupo control fue el mismo que para el grupo de padres de pacientes.

\section{RESULTADOS}

Los resultados obtenidos al comparar ambos grupos en las variables estudiadas han sido los siguientes:

Cuando comparamos los resultados obtenidos en esquizotipia psicométrica, como se puede observar en la Tabla 1, los padres de los pacientes esquizofrénicos difieren significativamente en el factor de anhedonia introvertida del OLIFE. En el factor de desorganización cognitiva el grupo de padres de pacientes puntúa más alto aunque la diferencia no llega a la significación. En cuanto a los trastornos de personalidad evaluados mediante el Cuestionario de Evaluación del IPDE, los resultados muestran que el grupo de padres de pacientes con esquizofrenia puntúa significativamente más en los trastornos paranoide y evitativo de la personalidad. Para los otros trastornos no se obtuvieron diferencias significativas.

En cuanto a la psicopatología evaluada mediante el SCL-90-R los resultados muestran que existen diferencias significativas entre ambos grupos. Como se puede observar en la Tabla 2, los padres de los pacientes con esquizofrenia obtienen puntuaciones significativamente más altas en las escalas de obsesividadcompulsividad, sensibilidad interpersonal, depresión, ansiedad, hostilidad e ideación paranoide. En todas las otras escalas excepto en la de somatización, las puntuaciones aunque no llegan a la significación son más elevadas en el grupo de los padres de esquizofrénicos. En cuanto a las diferencias en las escalas adicionales del SCL-90 podemos observar que los padres de los pacientes muestran significativamente más pérdida del apetito, alteraciones del sueño (conciliación, mantenimiento y despertar prematuro) y más sentimientos de culpabilidad.

En cuanto a los índices generales que proporciona el SCL-90-R podemos observar en las Figuras 1 y 2 que el grupo de padres de pacientes difiere del grupo control en todos ellos.

Para el f́ndice de Sintomatología General la puntuación media del grupo de padres de pacientes esquizofrénicos es de $0,38( \pm 0,17)$, para el grupo control es de $0,27( \pm 0.18)$ y la diferencia entre ambos es significativa $(t=3,88 ; p<0,001)$. En el 
Tabla 1. Comparación de las puntuaciones en los factores de esquizotipia y en los trastornos de personalidad entre ambos grupos.

\begin{tabular}{|c|c|c|c|c|}
\hline & $\begin{array}{c}\text { Grupo padres } \\
\text { pacientes } \\
(n=52) \\
M \pm D T \\
\end{array}$ & $\begin{array}{c}\text { Grupo padres } \\
\text { control } \\
(n=52) \\
M \pm D T \\
\end{array}$ & $t$ & $\begin{array}{c}\text { Significación } \\
p \leq\end{array}$ \\
\hline Experiencias inusuales & $3,37 \pm 2,84$ & $3,90 \pm 3,54$ & $-0,855$ & 0,395 \\
\hline Desorganización cognitiva & $9,77 \pm 4,03$ & $8,27 \pm 4,05$ & 1,893 & 0,061 \\
\hline Anhedonia introvertida & $10,50 \pm 4,08$ & $8,00 \pm 3,07$ & 3,534 & 0,001 \\
\hline Impulsividad no conformista & $3,27 \pm 2,26$ & $2,73 \pm 2,07$ & 1,268 & 0,208 \\
\hline Paranoide & $2,60 \pm 1,19$ & $2,02 \pm 1,36$ & 2,296 & 0,024 \\
\hline Esquizoide & $2,42 \pm 0,89$ & $2,10 \pm 1,11$ & 1,657 & 0,101 \\
\hline Esquizotípico & $1,38 \pm 0,95$ & $1,31 \pm 1,00$ & 0,401 & 0,689 \\
\hline Histriónico & $1,42 \pm 1,26$ & $1,77 \pm 1,13$ & $-1,476$ & 0,143 \\
\hline Antisocial & $0,83 \pm 0,83$ & $0,81 \pm 0,99$ & 0,107 & 0,915 \\
\hline Narcisista & $1,37 \pm 1,39$ & $1,73 \pm 1,21$ & $-1,434$ & 0,155 \\
\hline Límite & $2,79 \pm 1,18$ & $2,54 \pm 1,02$ & 1,158 & 0,250 \\
\hline Obsesivo-compulsivo & $2,17 \pm 1,72$ & $1,77 \pm 1,17$ & 1,400 & 0,165 \\
\hline Dependiente & $2,71 \pm 1,19$ & $2,63 \pm 0,91$ & 0,370 & 0,712 \\
\hline Evitativo & $2,46 \pm 0,94$ & $2,10 \pm 0,82$ & 2,111 & 0,037 \\
\hline
\end{tabular}

Tabla 2. Comparación del nivel de gravedad en las escalas del SCL-90 entre ambos grupos.

\begin{tabular}{lcccc}
\hline & $\begin{array}{c}\text { Grupo padres } \\
\text { pacientes } \\
(\mathrm{n}=52) \\
\mathrm{M} \pm \mathrm{DT}\end{array}$ & $\begin{array}{c}\text { Grupo padres } \\
\text { control } \\
(\mathrm{n}=52) \\
\mathrm{M} \pm \mathrm{DT}\end{array}$ & $t$ & $\begin{array}{c}\text { Significación } \\
p \leq\end{array}$ \\
\hline Somatización & $0,55 \pm 0,35$ & $0,61 \pm 0,30$ & $-1,016$ & 0,312 \\
Obsesividad-compulsividad & $0,36 \pm 0,37$ & $0,24 \pm 0,26$ & 2,000 & 0,048 \\
Sensibilidad interpersonal & $0,33 \pm 0,34$ & $0,17 \pm 0,16$ & 3,208 & 0,002 \\
Depresión & $0,49 \pm 0,31$ & $0,30 \pm 0,19$ & 3,664 & 0,000 \\
Ansiedad & $0,61 \pm 0,31$ & $0,44 \pm 0,25$ & 3,060 & 0,003 \\
Hostilidad & $0,27 \pm 0,30$ & $0,13 \pm 0,19$ & 2,825 & 0,006 \\
Ansiedad fóbica & $0,11 \pm 0,18$ & $0,08 \pm 0,12$ & 0,900 & 0,370 \\
Ideación paranoide & $0,38 \pm 0,39$ & $0,22 \pm 0,28$ & 2,483 & 0,015 \\
Psicoticismo & $0,10 \pm 0,14$ & $0,07 \pm 0,09$ & 1,501 & 0,136 \\
Apetito negativo & $0,38 \pm 0,79$ & $0,11 \pm 0,43$ & 2,149 & 0,034 \\
Apetito positivo & $0,19 \pm 0,59$ & $0,19 \pm 0,56$ & 0,000 & 1,000 \\
Alteraciones del sueño & & & & \\
Conciliación & $0,56 \pm 0,83$ & $0,31 \pm 0,51$ & 1,860 & 0,066 \\
Despertar prematuro & $0,73 \pm 1,21$ & $0,23 \pm 0,55$ & 2,722 & 0,008 \\
$\quad$ Mantenimiento & $0,63 \pm 0,89$ & $0,23 \pm 0,51$ & 2,849 & 0,005 \\
Pensamientos de muerte & $0,11 \pm 0,32$ & $0,10 \pm 0,30$ & 0,316 & 0,753 \\
Culpabilidad & $0,19 \pm 0,40$ & $0,09 \pm 0,14$ & 2,962 & 0,004 \\
\hline
\end{tabular}

índice del Nivel de Alteración de los Síntomas Positivos, la media de la puntuación de los padres de pacientes esquizofrénicos es de $1,49( \pm 0,31)$ y la del grupo control de $1,31( \pm 0,24)$, con una diferencia significativa del $0,002(t=3,209)$. La media del Total de Síntomas Positivos para el grupo índice es de $22,87( \pm 8,87)$ y para el grupo control de $18,17( \pm 6,63)$ $(t=3,055 ; p \leq 0,003)$.

Finalmente los resultados en las dimensiones del locus de control para la salud evaluado mediante el MHLC, muestran que los padres de los pacientes 

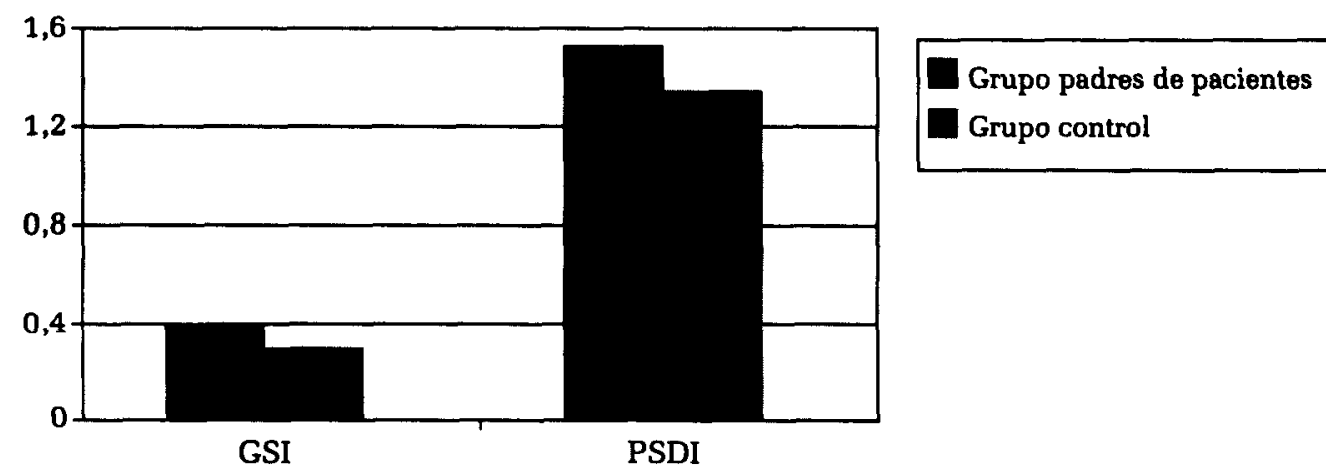

Figura 1. Puntuaciones en el f́ndice de Sintomatología General (GSI) y en el índice del Nivel de Alteración de los Síntomas Positivos del SCL-90-R (PSDI)

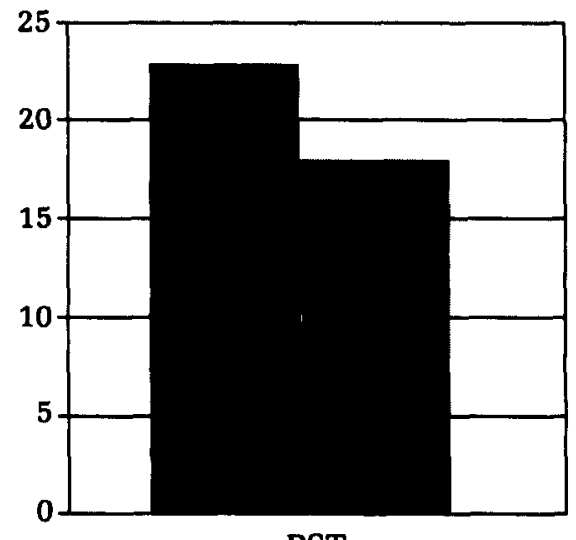

Grupo padres de pacientes

Grupo control

PST

Figura 2. Puntuaciones en el Total de Síntomas Positivos (PST)

con esquizofrenia puntúan más bajo en el locus de control interno (grupo de padres de pacientes $=14,67 \pm 5,23$; grupo de padres control $=16,98 \pm 4,58 ; p \leq 0,018)$ y en el poder de los otros (grupo de padres de pacientes $=18,94 \pm 4,87$; grupo de padres control $=21,06 \pm 5,20 ; p \leq 0,035$ ), es decir poseen una mayor creencia del control interno del reforzamiento relacionado con su propia salud, y mayor creencia en el poder de los otros, profesionales de la salud y familiares. No existen diferencias significativas en el factor suerte entre ambos grupos, aunque las medias indican una mayor tendencia en el grupo control a la creencia de que la salud depende sobre todo del azar (grupo de padres de pacientes $=18,83 \pm 6,15$; grupo de padres control $=17,35 \pm 4,97 ; p=0,180$ )

\section{DISCUSIÓN}

El objetivo principal de este estudio era la identificación de rasgos de vulnerabilidad a la esquizofrenia en padres no afectados de pacientes con esquizofrenia, es decir, en sujetos que ya han sobrepasado la edad de riesgo para el trastorno. Es importante señalar que ningún sujeto de los que han participado en el estudio ha sido tratado ni diagnosticado por ningún trastorno mental.

Como hemos podido observar en los resultados anteriores los padres de los pacientes con esquizofrenia presentan más rasgos de esquizotipia negativa que el grupo control. Los padres del primer grupo muestran más rasgos de anhedonia introvertida, es decir, mayor incapacidad 
para sentir placer y disfrutar de las situaciones sociales y de otras actividades, así como mayor aversión a la intimidad emocional y física y mayor tendencia a la soledad. Estos hallazgos están en consonancia con los hallados por otros autores, que afirman que los familiares de los pacientes esquizofrénicos evidencian con mayor probabilidad síntomas negativos (Lenzenwerger y Dworkin, 1984); así mismo, apoya la idea apuntada por Meehl $(1962,1989)$ de que la anhedonia es una consecuencia de un defecto genético.

En la línea de los resultados hallados por Grove et al. (1991), encontramos en nuestro estudio, una tendencia de los padres del grupo índice a describir más dificultades en mantener la atención, en concentrarse, en tomar decisiones y también en presentar más alteraciones del humor y más ansiedad social, como lo muestran las puntuaciones más elevadas (no siendo la diferencia significativa) de la dimensión de desorganización cognitiva.

Respecto a los rasgos positivos de la esquizotipia, evaluados mediante la dimensión de experiencias inusuales del O-LIFE, se puede observar que no sólo éstos no contribuyen a identificar a los padres de pacientes esquizofrénicos, sino que son los padres del grupo control los que obtienen mayores puntuaciones (aunque la diferencia entre los grupos no es significativa). Estos resultados no apoyan la hipótesis planteada, ya que, sorprendentemente, se hallan en la dirección opuesta a la esperada. Aunque los rasgos positivos se han identificado como rasgos de vulnerabilidad al trastorno, en el estudio de Clementz et al. (1991) también encontraron que en los familiares no afectados de sujetos esquizofrénicos la percepción aberrante era más baja que en los familiares afectados (con diagnósticos relacionados a la esquizofrenia) y que en los sujetos controles. Estos datos pueden sugerir que los síntomas positivos de la esqui- zotipia no identifican a los sujetos normales con vulnerabilidad, sino que se hallan más unidos a una manifestación más cercana a los trastornos relacionados con la esquizofrenia.

Si nos remitimos a las características que definen la anhedonia introvertida, podemos observar que las características comportamentales se dirigen básicamente a la evitación de situaciones que pueden ser potencialmente estresantes, y más concretamente, en el caso de padres de pacientes con una enfermedad todavía hoy en día estigmatizada socialmente. Quizás al evitar estas situaciones, estos sujetos reduzcan el estrés y esto propicie que exista un mayor control de los síntomas que pueden ser más graves y distorsionadores. Se podría pensar entonces, que la anhedonia introvertida es un rasgo de vulnerabilidad que "protege», a los sujetos que la presentan, de situaciones estresantes que puedan provocar la aparición de alteraciones más manifiestas de la esquizotipia o de trastornos relacionados con la esquizofrenia.

Respecto a los resultados referentes a los trastornos de personalidad podemos observar que los padres de los pacientes muestran más características del trastorno paranoide de la personalidad que los padres del grupo control, es decir, éstos presentan un mayor grado de sospecha y desconfianza hacia los demás, tendencia a vislumbrar intenciones amenazantes, afecto restringido y aplanado, etc. $\mathrm{Al}$ igual que en el estudio de Kendler y Gruenberg (1984) estos resultados indican que el trastorno paranoide de la personalidad forma parte de los trastornos del espectro esquizofrénico, dado que se presenta en mayor grado en los familiares de pacientes con esquizofrenia. Por otra parte, en nuestro estudio los resultados no muestran diferencias significativas entre ambos grupos en relación a la manifestación de más características de los otros dos trastornos del cluster A, 
trastorno esquizotípico y esquizoide de la personalidad. Otros autores, como Maier et al. (1994), también hallaron una baja proporción de trastorno esquizoide en familiares de esquizofrénicos, no revelando un poder estadístico suficiente para detectar diferencias entre grupos de familiares de pacientes con diversos trastornos mentales y controles. Respecto al trastorno esquizotípico de la personalidad, la literatura ha mostrado que éste está altamente ligado a la esquizofrenia (Gherson et al., 1988; Onstad et al., 1991; Maier et al., 1994), pero nuestros resultados no apoyan este hallazgo. Se debe tener en cuenta que en los estudios realizados los sujetos eran mayoritariamente familiares de pacientes esquizofrénicos, en los que se podían encontrar tanto padres como hijos como hermanos, es decir, parte de los sujetos con probabilidad todavía de presentar el trastorno. En nuestro caso, puede que las características de los sujetos estudiados, padres no afectados con poca probabilidad de que manifiesten ya el trastorno, expliquen el hecho de no hallar más características de trastorno esquizotípico de la personalidad. El trastorno esquizotípico por sus características definitorias, está más cercano al fenotipo esquizofrénico que los otros trastornos, y los padres que han formado parte del estudio no parece que hayan presentado nunca alteraciones tan cercanas al trastorno, al menos nunca han sido ni diagnosticados ni tratados por ellas. Así, pues, puede que las características específicas del trastorno esquizotípico de la personalidad sean poco manifiestas en sujetos familiares de esquizofrénicos de edades avanzadas y, por tanto, con poca probabilidad de presentar nunca el trastorno. Por otra parte, debemos ser conscientes que la muestra objeto de estudio puede estar sesgada, ya que, posiblemente, los padres que accedieron voluntariamente a formar parte de él sean los más «normales", y quedasen autoexcluidos los padres, aunque no afectados, con más vulnerabilidad.

En cuanto a la manifestación de otras características de los trastornos de personalidad hemos podido observar que los padres de los pacientes muestran puntuaciones más elevadas en el trastorno por evitación de la personalidad, trastorno no relacionado en la literatura con la esquizofrenia ni con la vulnerabilidad de ésta. Analizando las características definitorias de este trastorno, podemos observar que éste se caracteriza por un patrón de inhibición social y de hipersensibilidad a la evaluación negativa por parte de otras personas. No es sorprendente entonces, que los padres de los pacientes muestren más rasgos de este tipo, ya que están relacionados con las características más manifiestas de la anhedonia social y puede que también con ciertos rasgos de personalidad paranoide.

En cuanto a la psicopatología referida a la presentación de diversos síntomas en la semana previa a la evaluación (así pues, características de estado y no a rasgos permanentes de los sujetos), los resultados muestran que los padres de los pacientes puntúan significativamente más alto que el grupo control en la mayoría de las escalas. Los primeros muestran más sentimientos de tristeza, de desesperanza, de ansiedad, más nerviosismo, temor a que algo malo pueda suceder; reaccionan más sensiblemente los comentarios y críticas de otras personas; muestran más irritabilidad y hostilidad hacia los otros; también presentan más características obsesivas-compulsivas, es decir, comprueban más, tardan más tiempo en realizar diferentes actividades, se fijan más en los detalles, etc., y presentan más ideación paranoide. De la misma manera presentan más alteraciones del sueño e informan de pérdida del apetito. Todas estas alteraciones están relacionadas con los síntomas de ansiedad y depresión. Los padres de los 
pacientes también refieren más sentimientos de culpabilidad. Todas estas características, aunque no llegan a la patología, se muestran con mayor frecuencia en los padres de los pacientes con esquizofrenia que en el grupo control. Es fácil pensar que muchas de estas manifestaciones (como por ejemplo los síntomas de depresión y ansiedad) se deben a una reacción ante la situación estresante de tener un hijo con esquizofrenia, situación que tiene importantes repercusiones negativas en los padres que la padecen. Algunas de las otras manifestaciones pueden deberse a un mecanismo de afrontamiento, como es el caso de la sensibilidad interpersonal, la hostilidad y la ideación paranoide.

En cuanto a la variable locus de control hipotetizábamos que los padres de pacientes con esquizofrenia presentarían un menor locus de control interno de acuerdo con el estudio de Böker et al. (1989). A partir de los resultados obtenidos vemos que esto no se cumple. En nuestro estudio los padres del grupo índice obtienen puntuaciones más bajas en la dimensión de «internalización», es decir, las puntuaciones se dirigen hacia un LOC significativamente más interno que en los padres del grupo control. Los padres de los pacientes atribuyen un mayor peso al control interno en relación al refuerzo del propio estado de salud que los padres del grupo control, es decir, piensan que el estado de salud depende en parte del control que ellos tienen sobre ésta. Analizando más detalladamente los resultados de Böker et al. (1989), podemos observar que éstos realizan una diferenciación entre aquellos familiares de esquizofrénicos con vulnerabilidad al trastorno y familiares sin vulnerabilidad al trastorno; el criterio de vulnerabilidad que utilizan es el tiempo de reacción. Realizando esta diferenciación, hallan que los familiares con vulnerabilidad muestran un menor LOC interno que los otros. En nuestro estudio no se ha realizado esta diferenciación, pero teniendo en cuenta que los padres eran sujetos no afectados, podemos pensar que existe una equiparación con sujetos con una "menor vulnerabilidad" y entonces, los resultados no se hallan tan lejos de los encontrados por estos autores. Por otra parte, la creencia de control interno facilita la adaptación del individuo a situaciones estresantes y posiblemente, los padres de los pacientes esquizofrénicos utilicen este mecanismo para enfrentarse a la complicada situación de tener un hijo enfermo, ya que, presumiblemente, las creencias de control interno están relacionadas con estrategias de afrontamiento más adaptativas. Respecto al LOC externo "poder de los otros», también los padres de los pacientes muestran una mayor creencia de que otras personas (sobre todo profesionales del campo de la salud) tienen un peso importante en el mantenimiento de la propia salud. Este resultado es fácilmente comprensible si tenemos en cuenta que estas personas están continuamente en contacto con médicos psiquiatras y psicólogos que tratan el trastorno de sus hijos y la importancia que estos contactos poseen para el tratamiento y mejora de la esquizofrenia. El LOC «poder de los otros» es una dimensión conceptualizada como externa, ya que no es el propio control el que ejerce un efecto en el refuerzo relacionado con la salud, sino que son otras personas «externas» al propio sujeto las que poseen este control. Podemos pensar, no obstante, que existe una buena parte de control interno en esta dimensión, ya que es la propia persona la que va en busca y recibe la ayuda de otros profesionales, y esto está ligado a la creencia de que uno mismo sí puede hacer algo por su propia salud. Respecto al LOC "suerte», aunque los padres de los pacientes le otorgan un menor peso que los del grupo control, las diferencias no son significativas, es decir 
ambos grupos piensan de manera similar que la suerte juega un papel importante en relación a la salud. En resumen, los padres no afectados de pacientes esquizofrénicos manifiestan mayores creencias de que el control interno y el poder de los otros juegan un importante papel en la salud.

Para finalizar podemos decir que, posiblemente, las características de vulnerabilidad manifiestas en un fenotipo de personalidad en personas unidas genéticamente a pacientes con esquizofrenia, se presentan de manera muy diversa. Existen sujetos en los que estas características se manifiestan de tal manera que pueden ser evaluadas y medidas, pero seguramente, existen también sujetos, familiares de esquizofrénicos, en los que es más complejo afirmar que estas características de personalidad si se dan, son rasgos de vulnerabilidad subyacente al trastorno, y sujetos en los que, por su "normalidad", estas características alejadas del "patrón de normalidad" no se manifiesten.

\section{REFERENCIAS BIBLIOGRÁFICAS}

Böker, W., Brenner, H.D., y Würgler, S. (1989). Vulnerable-linked deficiencies, psychopatho-logy and coping behaviour of schizophrenics and their relatives. British Journal of Psychiatry, 155, 128-135.

Brenner, H.D., Böker, W., Müller, J., y Spichtig (1987.) On autoprotective efforts of schizophrenics, neurotics and controls. Acta Psychiatrica Scandinavica, 75, 405414.

Cardno, A. G., y McGuffin, P. (1996). Aetiological theories of schizophrenia. Current Opinion in Psychiatry, 9, 45-49.

Cash, T.F., y Stack, J.J. (1973). Locus of control among schizophrenic and other hospitalised psychiatric patients. Genetic Psychology Monographs, 87, 105-122.

Clementz, B.A., Grove, W.M., Katsanis, J., y Iacono, W. (1991). Psychometric detection of schizotypy: perceptual aberration and physical anhedonia in relatives of schizophrenics. Journal of abnormal Psychology, 100, 607-612.

Derogatis, L. (1977). SCL-90-R: Administration, Scoring and Procedure Manual I. Baltimore, (MD): Clinical Psychometric Research.

Enright, S., y Beech, A. (1997). Schizotypy and obsessive-compulsive disorder. En: Claridge, G. (ed.) Schizotypy. Implications for illness and health, 203-223. Oxford: Oxford University Press.

Erlenmeyer-Kimling, L., Squires-Wheeler, E., Adamo, U. H., Bassett, A. S., Cornblatt, B. A., Kestenbaum, C.J., Rock, D.; Roberts, S.A., y Gottesman, I.I. (1995). The New York High-Risk Project: Psychoses and cluster A personality disorders in offspring of schizophrenic parents at 23 years of follow-up. Archives of General Psychiatry, 52, 857-865.

Evans, D.R. (1997). Health promotion, wellness programs, quality of life ant the marketing of psychology. Canadian Psychology, 38, 1-12.

Gershon, J.M., DeLisi, L.E., Hamovit, J., Nurnberger, J.I., Maxwell, M.D., Schreiber, J., Dauphinais, D., Dingman, C.W., y Guroff, J.J. (1988). A controled family study of chronic psychoses. Archives of General Psychiatry, 45, 328-336.

González de Rivera, J.L., Derogatis, L.R., de las Cuevas, C., Gracia Marco., R., Rodríguez-Pulido, F., Henry-Benitez, M., y Monterrey, A.L. (1989). The spanish version of the SCL-90-R. Normative data in the general population. Towson, Clinical Psychometric Research.

Grove, W.M., Lebow, B.S., Clementz, B.A., Cerri, A., Medus, C., y Iacono, W.G. (1991). Familial prevalence and coaggregation of schzizotypy indicators: a multitrait family study. Journal of Abnormal Psychology, 2, 115-121.

Gutiérrez, J., Aguilar, A., Aguiler, E.M., Barrantes-Vidal, N., y Obiols, J.E. (1998). Adaptación española reducida de las escalas O-LIFE. Póster presentado en el V Congreso de Evaluación Psicológica. Málaga, mayo 1998.

Holzman, P.S. (1982). The search for a biological marker of the functional psychoses. 
En: Goldstein, M.J. (Ed.), Preventive intervention in schizophrenia: Are we ready? (pp.19-38). Washington, D.C.: U.S. Government Printing Office.

Kendler, K.S., y Gruenberg, A.M. (1984). An independent analysis of the Danish Adoption Study of schizophrenia: VI. The relationship between psychiatric disorders as defined by DSM-III in the relatives and adoptees. Archives of Genral Psychiatry, 41, 555-564.

Kendler, K.S., Masterson, C.C., Ungaro, R., y Davis, K.L. (1984). A family history study of schizophrenia related personality disorders. American Journal of Psychiatry, 141, 424-427.

Kendler, K.S., Gruenberg A.M., y Tsuang, M.T. (1985). Psychiatric illness in firstdegree relatives of schizophrenic and surgical control patients: a family study using DSM-III criteria. Archives of General Psychiatry, 42, 770-779.

Kendler, K.S, Ochs, A.L., Gorman, A.M., Hewitt, J.K., Ross, D.E., y Mirsky, A.F. (1991). The structure of schizoypy: a pilot multitrait twin study. Psychiatry Research, 36, 19-36.

Kendler, K.S, McGuire, M., Gruenberg, A.M., O'Hare, A., Spellman, M., y Walsh, D. (1993). The Roscommon family study. IV. Affective illness, anxiety disorders and alcoholism in relatives. Archives of General Psychiatry, 50, 952-960.

Kety, S.S., Wender, P.H., Jacobsen, B., Ingraham, L.J., Janson, L., Faber, B., y Kinney, D.K. (1994). Mental illness in the biological and adoptive relatives of schizophrenic adptees. Replication of the Copenhagen Study in the rest of Denmark. Archives of Genral Psychiatry, 51, 442-455.

Levenson, H. (1973). Multidimensional locus of control in psychiatric patients. Journal of Consulting and clinical Psychology, 41, 397-404.

Lenzenweger, M.F., y Dworkin, R.H. (1984). Symptoms and the genetics of schizophrenia: implications for diagnosis. American Journal of Psychiatry, 141, 1541-1546.

Lenzenweger, M.F., y Loranger, A.W. (1989). Psychosis proneness and clinical psychopa-thology: Examination of the correlayes of schizotypy. Journal of Abnormal Psychology, 98, 3-8.
Loranger, A.W. (1988). Personality Disorder Examination (PDE) Manual.Yonkers, NY: DV Communications.

Maier, W., Lichtermann, D., Minges, J., y Reinhard, H. (1994). Personality disorders among the relatives of schizophrenia patients. Schizophrenia Bulletin, 20, 481493.

Mason, O., Claridge, G., y Jackson, M. (1995). New scales for the assessment of schizotypy. Personality and Individual Differences, 1, 7-13.

Marcus, J., Hans, S.L., Auerbach, J.G., y Auerbach, A.G. (1993). Children at risk for schizophrenia: the Jerusalem Infant Development Study. II. Neurobehavioral deficits at school age. Archives of General Psychiatry, 50, 797-809.

McGue, M., Gottesman, I.I., y Rao, D.C. (1986). The analysis of schizophrenia family data. Behavior Genetics, 16, 75-87.

Meehl, P.E. (1962). Schizotaxia, schizotypy, schizophrenia. American Psychologist, 17, 827-838.

Meehl, P.E. (1989). Schizotaxia revisited. Archives of General Psychiatry, 46, 935944.

Organización Mundial de la Salud (1996). I.P.D.E. Examen Internacional de los Trastornos de la Personalidad, Módulo DSMIV. Madrid: Meditor.

Onstad, S., Skre, I., Edvardsen, J., Togersen, S., y Kringlen, E. (1991). Mental disorders in first-degree relatives of schizophrenics. Acta Psychiatrica Scandinavica, 83, 463467.

Rado, S. (1953). Dynamicas and classification of disordered behavior. American Journal of Psychiatry, 110, 406-416.

Rotter, J.B., Chance, J.E., y Phares, E.J. (1972). Applications of a social learning theory of personality. New York: Holt, Rinehart and Winston.

Tienari, P., Lahti, I., Sorri, A., Naarala, M., Moring, J., y Wahlberg, K. (1989). The Finnish adoptive family study of Schizophrenia: Possible joint effects of genetic vulnerability and family environment. British Journal of Psychiatry, 155, 29-32.

Varma, S. L., Zain, A. M., y Singh, S. (1997). Psychiatric morbidity in the first-degree relatives of schizophrenic patients. 
American Journal of Medics Genetics, 74, 7-11.

Wallston, K.A., Wallston, B.S., y DeVellis, R. (1978). Development of the Multidimensional Health Locus of Control (MHLC) Scales. Health Education Monographs, 6, 160-171. Winokur, G., Scharfetter, C., y Angst, J. (1985). A family study of psychotic symp- tomatology in schizophrenia, schizoaffective disorder, unipolar depression and bipolar disorder. European Aechives of Psychiatry and Neurological Sciences, 234, 295-298.

Zubin J., y Spring, B.J. (1977). Vulnerability: A new view of schizophrenia. Journal of Abnormal Psychology, 86, 103-126. 